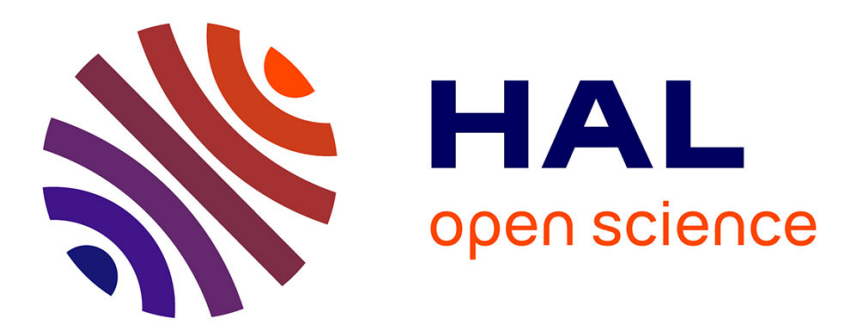

\title{
Segmentation of Rough Surfaces Using a polarization Imaging System
}

Patrick Terrier, Vincent Devlaminck, Jean-Michel Charbois

\section{To cite this version:}

Patrick Terrier, Vincent Devlaminck, Jean-Michel Charbois. Segmentation of Rough Surfaces Using a polarization Imaging System . Journal of the Optical Society of America. A Optics, Image Science, and Vision, 2008, 25 (2), pp.423- 430. hal-00648855

\section{HAL Id: hal-00648855 \\ https://hal.science/hal-00648855}

Submitted on 9 Mar 2012

HAL is a multi-disciplinary open access archive for the deposit and dissemination of scientific research documents, whether they are published or not. The documents may come from teaching and research institutions in France or abroad, or from public or private research centers.
L'archive ouverte pluridisciplinaire HAL, est destinée au dépôt et à la diffusion de documents scientifiques de niveau recherche, publiés ou non, émanant des établissements d'enseignement et de recherche français ou étrangers, des laboratoires publics ou privés. 


\title{
Segmentation of Rough Surfaces Using a Polarization Imaging
}

\section{System}

\author{
Patrick Terrier, Vincent Devlaminck, Jean Michel Charbois \\ Laboratoire LAGIS UMR CNRS 8146, Université des Sciences et Technologies de Lille. \\ Bâtiment P3, 59655 Villeneuve d'Ascq cedex, France \\ e-mail : patrick.terrier@univ-lille1.fr
}

\section{INTRODUCTION}

The conventional method for measuring surface roughness consists in moving a stylus probe touching the surface and monitoring its movement in order to reconstruct the surface microprofile. This technique has been developed to a sophisticated level [1]. However, using a stylus probe has several drawbacks: fragility of the sensing probe, surface damages caused by contact, etc.

On the other hand, laser optical methods, that do not require contact with the surface and allow for high measurement speed, have been widely used in industry[2,3]. In general, these methods are extremely accurate, but they yield measurements only for single point or small patch of the surface. Therefore, compared to vision methods, they are difficult to implement for on-line roughness quality control in manufacturing industries.

This paper deals with the implementation of a polarimetric vision based method yielding an image, i.e. pixel based estimation, of the roughness and the refractive index of an observed objects. Compared to previously published optical methods, based on light scattering[4], our goal is not to measure precisely the roughness, but to obtain information on 
the reflection properties of the surface allowing the segmentation of objects according to their roughness property. This segmentation is a first step towards the detection of surface defects.

Like several published works[5,6,7], our approach is based on the microfacet reflection model.

Most of these works assume that the diffuse component is described by the Lambert model which is an idealistic behavior. On the other hand, for a rough surface, the specular component of the BRDF is assumed to be composed of a set of micro planar surfaces that reflect the incident light in various directions. The angular distribution of the specular component of the reflected light, related to the statistic angular distribution of microfacets inclinations, is estimated. This statistical parameter leads to information about the surface roughness. In our approach, the difference comes from that we propose to measure the reflected light wave state of polarization. This approach allows us to estimate the roughness parameter without assumption on the model of the diffuse component. Diffuse-specular separation is neither required. 


\section{FORMAL DEFINITION OF THE MODEL}

The microfacet model assumes that the surface is formed by a collection of small planar patches, or microfacets, acting as specular reflectors. Each microfacet obeys the SnellDescartes' law of reflection and corresponds to a local plane of incidence defined by a normal unit vector $\vec{n}$ (cf. Fig.1). Therefore, the angle of reflection $\theta_{\mathrm{R}}^{\prime}$ of a light beam reflected by the microfacet is equal to its angle of incidence. All the microfacets orientations are assumed to be distributed around the global normal $\vec{N}$. The spherical coordinates notations for the microfacet model geometry illustrated Fig.1 are: $\alpha$ angle between the surface normal $\vec{N}$ and the microfacets's normal $\vec{n}, \theta_{\mathrm{i}}$ illumination zenith angle, $\theta_{\mathrm{R}}$ viewing zenith angle.

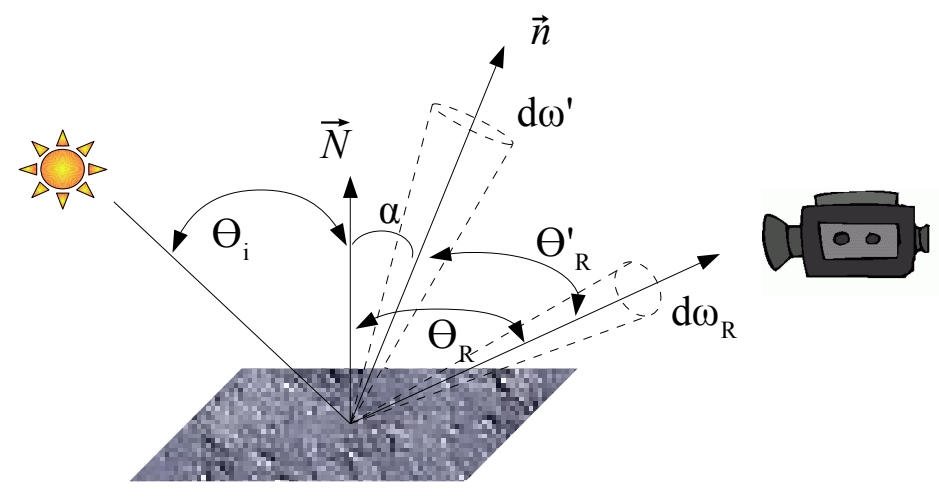

Fig. 1: Geometry of the microfacet model

The reflected light wave is composed of two reflection components, namely diffuse and specular. The diffuse component is the combination of light rays penetrating the surface, undergoing multiple reflections and refractions, and re-emerging at the surface. It is distributed in a wide range of directions around the surface normal $\vec{N}$, giving the surface a matte appearance. If the viewing direction $\theta_{R}$ varies while the angle of incidence $\theta_{\mathrm{i}}$ remains 
constant, the intensity of the diffuse component changes slowly, and even does not change at all in the ideal case of a Lambertian surface.

On the other hand, the specular component is the result of a surface phenomenon. Light rays reaching the surface are reflected by the microfacets that are aligned with the direction verifying Snell-Decartes's law: the angle of incidence $\theta_{\mathrm{i}}^{\prime}$ (between the illuminating direction and the microfacets's normal $\vec{n}$ ) is equal to the angle of reflection $\theta_{\mathrm{R}}^{\prime}$ (between the microfacets's normal and the viewing direction). One can notice here that the main assumption of this model is that the microfacets are much larger than the wavelength, allowing us to apply the geometric optic laws. Under this assumption the specular reflections are concentrated in a lobe around the specular direction, i.e. the direction of reflection off a mirror-like smooth surface with $\theta_{\mathrm{R}}=\theta_{\mathrm{i}}$.

Therefore, a reflected beam is considered as a linear combination of the diffuse reflection component, and the specular reflection component. The orientation of the specular component, which is related to the orientation of each microfacet, can be described by the distribution proposed by Cook and Torrance [8], with a probability density function which depends on a roughness parameter $\sigma$ (eq. (1)):

$$
D(\alpha)=\frac{1}{\sigma^{2} \cos ^{4} \alpha} \exp \left(-\frac{\tan ^{2} \alpha}{\sigma^{2}}\right)
$$

The spread of the orientations of specular components is related to the RMS slope $\sigma$. For small values of $\sigma$, i.e. when microfacets are almost parallel to the surface, the distribution is highly directional around the specular direction. For large values of $\sigma$, i.e. when microfacets have a very steep average slope, the distribution is spread out. In the geometry shown in fig.1, only planar microfacets having normal vectors $\vec{n}$ within a solid angle $\mathrm{d} \omega$ ' can reflect the 
incoming light flux specularly in a solid angle $\mathrm{d} \omega_{\mathrm{R}}$.

On the other hand the diffuse component is generally described by the Lambert model which assumes that light is isotropically reflected, this is, of course, an idealistic behavior.

Consequently the intensity measured on a pixel of the image is generally modeled, in computer vision literature, by the following relation (see ref. [9] for details):

$$
I_{C}=K_{D, C} \cos \theta_{i}+\frac{K_{S, C} F\left(\eta, \theta^{\prime}{ }_{i}\right) G\left(\alpha, \theta^{\prime}{ }_{i}, \theta_{i}, \theta_{R}\right) D(\alpha)}{\cos \theta_{R}}
$$

where the index $C$ represents a color component (R, G, B for instance), $K_{D, C}$ and $K_{S, C}$ are respectively the coefficients for the diffuse and specular reflection components, $F\left(\eta, \theta_{i}^{\prime}\right)$ is the Fresnel reflection coefficient (depending on the refractive index $\eta$ of the material) and $G\left(\alpha, \theta_{i}^{\prime}, \theta_{i}, \theta_{R}\right)$ is the geometric attenuation factor. The term $G$ accounts for the fact that, for large incidence angles, the light reaching a facet may be shadowed by adjacent surface irregularities, and that the outgoing light along the viewing direction that grazed the surface can be masked on its path to the viewer. This term is given by:

$$
G=\min \left\{1 ; \frac{2 \cos \alpha \cos \theta_{R}}{\cos \theta_{i}^{\prime}} ; \frac{2 \cos \alpha \cos \theta_{i}}{\cos \theta_{i}^{\prime}}\right\}
$$

Several published works[5,6,7] aimed to estimate unknown parameters $K_{D, C}, K_{S, C}$ and $\sigma$ with non-linear least square method by minimizing the squared difference between the left hand side and right hand side of equation (2). We can see according to this equation that the roughness parameter $\sigma$ may be estimated with less accuracy if in practice the diffuse component is not described by the idealistic Lambertian model [10]. Sometimes diffuse and 
specular component are beforehand separated using the dichromatic reflection model [11]. This model assumes that diffuse and specular components generally have different spectral distributions. Published methods $[12,13,14]$ generally yields good separation results but the iterative form of the derived algorithms and the constraint of neighboring pixels with similar diffuse color sometimes limit their applicability.

Therefore, in this paper we aim to estimate the roughness parameter $\sigma$ without requiring any assumption on the diffuse component. For this we use the fact that diffuse and specular components of reflection have different polarization states [15]. The diffuse component is generally assumed to be unpolarized while the specular component is assumed to be partially polarized. The polarization of the specular component is directly connected to the material constituting the observed object (by its refractive index $\eta$ ) and to its surface roughness $\sigma$. This will be explained more precisely in the following $\S$.

\section{MEASUREMENT DEVICE}

In our experiment we use a Stokes polarimeter [16] to measure the polarization state of the reflected light waves. The samples under test are illuminated by an incoherent light source, centered over the wavelength $\lambda_{0}=640 \mathrm{~nm}$. This source is constituted by a set of monochromatic led placed behind a diffusing screen in front of a linear $+45^{\circ}$ polarizer. The distance between the source and the samples is about half a meter.

The resulting light is analysed by the polarimeter, based on the use of two liquid crystals variable retarders (LCVR) follow-ups of a linear polarizer. Each one of these optical components makes it possible to modify the polarization state of the incident light wave without requiring mechanical actions on the device (rotations). The variable parameters of the device are a couple of delays $\left(\delta_{1}, \delta_{2}\right)$. Theses delays are adjusted by the amplitude of a 
rectangular alternative voltage that is applied to the LCVR by the intermediary of a command interface board.

The light intensity is then measured by a 12 bits CCD camera of $640 \times 480$ pixels. Images are digitized and processed by an image analysis system that has a 12-bit frame buffer. The distance between the camera and the samples gives a pixel resolution of about 100 micrometers.

The influence of each one of optical components on the state of polarization of the light wave is modeled by the Stokes-Mueller formalism. The positioning of each optical component is chosen in order to obtain an optimal measurement configuration [17].

With this system, the polarization state is described by a four-components Stokes vector $\boldsymbol{S}=$ $\left[\mathrm{S}_{0}, \mathrm{~S}_{1}, \mathrm{~S}_{2}, \mathrm{~S}_{3}\right]^{\mathrm{T}}$ and the reflection on planar microfacets is modeled by a $4 * 4$ Mueller matrix [18]. This matrix is related to the complex Fresnel reflection coefficients [19] $\rho_{\mathrm{S}}$ (perpendicular to the plane of incidence) and $\rho_{\mathrm{P}}$ (parallel to the plane of incidence). In general case the first medium is air, so these coefficients are defined by the following equations, where $\theta_{i}^{\prime}$ and $\gamma$ are respectively the incident and refracted angles.

$$
\begin{gathered}
\rho_{S}=\frac{\cos \theta^{\prime}{ }_{i}-\eta \cos \gamma}{\cos \theta^{\prime}{ }_{i}+\eta \cos \gamma} \\
\rho_{P}=\frac{\eta \cos \theta^{\prime}{ }_{i}-\cos \gamma}{\cos \gamma+\eta \cos \theta^{\prime}{ }_{i}}
\end{gathered}
$$

The refracted angle $\gamma$ is connected to the incidence angle $\theta_{i}^{\prime}$ and to the refractive index $\eta$ of the observed material by the Snell-Descartes' law of refraction (in the case of metallic objects $\eta$ is 
a complex number) .

$$
\cos \gamma=\frac{\sqrt{\eta^{2}-\sin ^{2} \theta^{\prime}}{ }_{i}}{\eta}
$$

Therefore, the Mueller matrix relation between the Stokes vector of the incident beam ant the Stokes vector of the specular reflected beam can be written as:

$$
M=\frac{1}{2}\left[\begin{array}{cccc}
\rho_{S} \rho_{S}^{*}+\rho_{P} \rho_{P}^{*} & \rho_{S} \rho_{S}^{*}-\rho_{P} \rho_{P}^{*} & 0 & 0 \\
\rho_{S} \rho_{S}^{*}-\rho_{P} \rho_{P}^{*} & \rho_{S} \rho_{S}^{*}+\rho_{P} \rho_{P}^{*} & 0 & 0 \\
0 & 0 & \rho_{S} \rho_{P}^{*}+\rho_{P} \rho_{S}^{*} & -j\left(\rho_{S} \rho_{P}^{*}-\rho_{P} \rho_{S}^{*}\right) \\
0 & 0 & j\left(\rho_{S} \rho_{P}^{*}-\rho_{P} \rho_{S}^{*}\right) & \rho_{S} \rho_{P}^{*}+\rho_{P} \rho_{S}^{*}
\end{array}\right]
$$

In the experimental configuration, the polarimeter is fixed in the vertical reference direction, a linear $+45^{\circ}$ polarized light source $\left(\boldsymbol{S}=\left[\mathrm{S}_{0}, 0, \mathrm{~S}_{0}, 0\right]^{\mathrm{T}}\right)$ is positioned at different directions $\left(\theta_{\mathrm{S}}, \varphi_{\mathrm{S}}\right)$, and the surface is oriented in a known direction $\left(\theta_{\mathrm{N}}, \varphi_{\mathrm{N}}\right)$, as illustrated in fig.2.

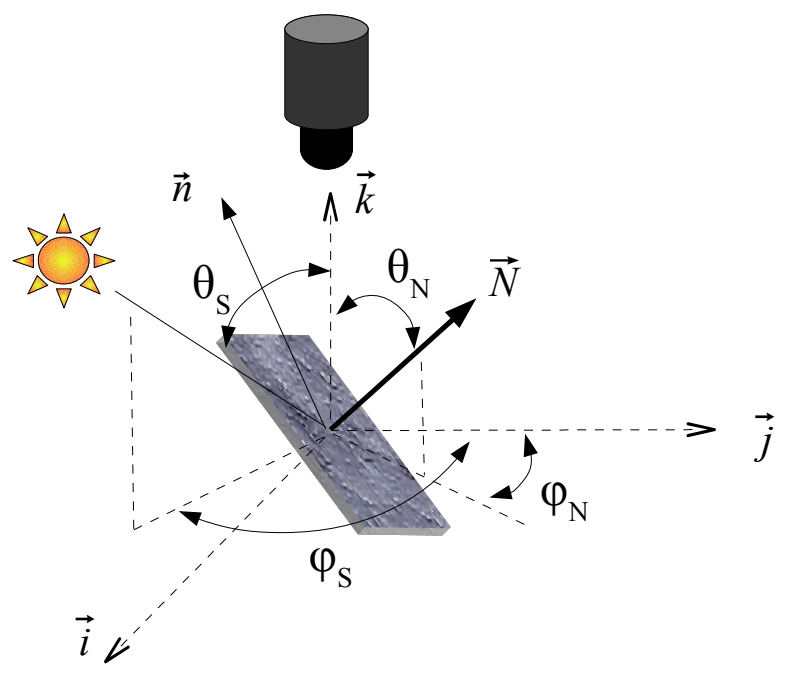

Fig.2: Geometry of the measurement device 
In the general case, when the measurement device observes a scene, each imaged object has an unknown random orientation. Here, we make the assumption that the orientations of objects' facets are estimated beforehand, for example by a stereo-vision technique [20].

In this configuration, all the angles $\left(\alpha, \theta_{\mathrm{R}}, \theta_{\mathrm{i}}, \theta_{\mathrm{i}}^{\prime}\right)$ defining the microfacet model (fig. 1$)$ can be estimated using spherical trigonometry (see appendix):

$$
\begin{aligned}
& \cos \alpha=\cos \theta_{N} \cos \frac{\theta_{S}}{2}+\sin \theta_{N} \sin \frac{\theta_{S}}{2} \cos \left(\varphi_{S}-\varphi_{N}\right) \\
& \theta_{R}=\theta_{N} \\
& \cos \theta_{i}=\cos \theta_{N} \cos \theta_{S}+\sin \theta_{N} \sin \theta_{S} \cos \left(\varphi_{S}-\varphi_{N}\right) \\
& \theta^{\prime}{ }_{i}=\frac{\theta_{S}}{2}
\end{aligned}
$$

The Stokes vector $\boldsymbol{S}^{\prime}$, measured by the polarimeter, corresponds to the contribution of the specular component of reflection (the Stokes vector $\boldsymbol{S}_{S}{ }_{S}$ is modeled by the Mueller formalism $\boldsymbol{S}_{S}^{\prime}=\boldsymbol{M S}$ ), plus the diffuse component of reflection $\boldsymbol{S}_{{ }_{\boldsymbol{D}}}^{\prime}$ that is assumed to be unpolarized $[14,15]\left(\boldsymbol{S}^{\prime}=\boldsymbol{S}_{\boldsymbol{D}}^{\prime}+\boldsymbol{S}_{\boldsymbol{S}}^{\prime}\right)$. According to eq.(1), (2) and (7), these two contributions are formally obtained, for a linear $+45^{\circ}$ polarized light source, by the relations:

$$
S_{D}^{\prime}=\left|\begin{array}{c}
S^{\prime}{ }_{0 \mathrm{D}} \\
0 \\
0 \\
0
\end{array}\right|=\left|\begin{array}{c}
K_{D} \cos \theta_{i} \\
0 \\
0 \\
0
\end{array}\right|\left|\begin{array}{c}
S_{0} \\
0 \\
0 \\
0
\end{array}\right|
$$




$$
S_{S}^{\prime}=\left|\begin{array}{l}
S^{\prime}{ }_{0 \mathrm{~S}} \\
S^{\prime}{ }_{1 \mathrm{~S}} \\
S^{\prime}{ }_{2 \mathrm{~S}} \\
S^{\prime}{ }_{3 \mathrm{~S}}
\end{array}\right|=K_{S} G \frac{1}{\sigma^{2} \cos ^{4} \alpha \cos \theta_{R}} \exp \left(-\frac{\tan ^{2} \alpha}{\sigma^{2}}\right)\left|\begin{array}{l}
S_{0} M_{11} \\
S_{0} M_{21} \\
S_{0} M_{33} \\
S_{0} M_{43}
\end{array}\right|
$$

where the coefficients $\mathrm{M}_{\mathrm{ij}}$ corresponds to the Mueller matrix characterizing the light reflection on planar microfacets. These coefficients depend on the refractive index $\eta$ of the material and on the angle $\theta_{\mathrm{i}}^{\prime}$ between the incident light beam and the local surface vector $\vec{n}$ (eq. 4,5,6). $\mathrm{S}_{0}$ corresponds to the intensity of the light source that is linearly polarized, and may be normalized to unity $\left(\mathrm{S}_{0}=1\right)$.

In our experiment, the measurement protocol is composed of four steps:

a) Make several measurements of the reflected optical light wave Stokes vector $\boldsymbol{S}^{\prime}$ for various positions of the light source.

b) Use the polarization information $\left(\mathrm{S}_{1 \mathrm{~S}}, \mathrm{~S}_{2 \mathrm{~S}}\right)$ of the specular Stokes vectors for estimating the refractive index $\eta$ of the observed material.

c) Estimate the value of the Mueller coefficients $M_{i j}$ for each position of the light source.

d) Use the specular component of reflection to estimate the roughness parameter $\sigma$ for each pixel of the observed scene. 


\section{ESTIMATION OF THE MATERIAL'S REFRACTIVE INDEX $\eta$}

As we can see on eq. (12) and (13) the polarized part $\left(\boldsymbol{S}_{1.33}^{\prime}\right)$ of the measured Stokes vector $\boldsymbol{S}^{\prime}$ is only related to the specular component of reflection. Therefore, the polarimetric measurements can be used to estimate the roughness parameter $\sigma$ of the observed surface according to eq. (13). However, this equation depends on the Mueller matrix coefficients $\mathrm{M}_{21}$, $\mathrm{M}_{33}$ and $\mathrm{M}_{43}$ characterizing the surface, which in their turn depend on the unknown refractive index $\eta$ of the material. It is thus necessary to estimate $\eta$ in a first step. For this, the measured Stokes parameter $\mathrm{S}_{2}{ }_{2}$ (equal $\mathrm{S}_{2 \mathrm{~S}}$ ) is divided by the Stokes parameter $\mathrm{S}_{1}{ }_{1}$ (equal $\mathrm{S}_{1 \mathrm{~S}}$ ) for each position of the light source. According to eq. (13) we have:

$$
\frac{S^{\prime}{ }_{2 \mathrm{~S}}}{S^{{ }_{1 \mathrm{~S}}}}=\frac{M_{33}}{M_{21}}=\frac{\rho_{S} \rho_{P}^{*}+\rho_{P} \rho_{S}^{*}}{\rho_{S} \rho_{S}^{*}-\rho_{P} \rho_{P}^{*}}=f\left(\eta, \theta^{\prime}{ }_{i}\right)
$$

The angles $\theta_{\mathrm{i}}^{\prime}$ are known for each position of the light source (eq. 11), thus the refractive index $\eta$ can be estimated by minimizing the merit function defined as:

$$
\Gamma=\sum_{\theta_{i}^{\prime}}\left(\left(\frac{S_{2 \mathrm{~S}}^{\prime}}{S^{\prime}{ }_{1 \mathrm{~S}}}\right)_{\text {mesured }}-f(\eta)_{\text {modeled }}\right)^{2}
$$

The refraction index $\eta$ is then used to compute the coefficients $M_{i j}$ for each position of the light source. As a consequence, all parameters of eq. (13) are known, except $\sigma$ and Ks. 


\section{ESTIMATION OF SURFACE ROUGHNESS}

In order to solve eq. (13) for the two unknowns $\sigma$ and Ks, we linearize the equation by a logarithmic transformation and obtain:

$$
\ln \left(\frac{S^{\prime}{ }_{1 \text { or } 2 S} \cos ^{4} \alpha \cos \theta_{R}}{G M_{21 \text { or } 33}}\right)=\ln \left(\frac{K_{S}}{\sigma^{2}}\right)-\frac{\tan ^{2} \alpha}{\sigma^{2}}
$$

For $\mathrm{N}$ positions of the light source, we have $2 \mathrm{~N}$ points: one point per position and one per Stokes parameter. The coordinates of each point for a given position of the source are:

$$
\begin{aligned}
& Y_{1}=\ln \left(\frac{S^{\prime}{ }_{1 \mathrm{~S}} \cos ^{4} \alpha \cos \theta_{R}}{G M_{21}}\right) \\
& Y_{2}=\ln \left(\frac{S^{\prime}{ }_{2 \mathrm{~S}} \cos ^{4} \alpha \cos \theta_{R}}{G M_{33}}\right) \\
& X=\tan ^{2} \alpha
\end{aligned}
$$

Using a least-squares method, we fit a line on these $2 \mathrm{~N}$ points, which yields an estimation of the slope $-1 / \sigma^{2}$ of the linear model of equation (16), therefore an information about surface roughness for each pixel of the image.

Note that this estimation of $\sigma$ can be made for different color bands $\left(\mathrm{K}_{\mathrm{S}}\right.$ being different for each color but not $\sigma$ ). So, instead of using $2 \mathrm{~N}$ points (one point per Stokes parameter and per position of the light source) we can use $6 \mathrm{~N}$ points for images with three color channels, which yields a more precise estimation of $\sigma$. 


\section{EXPERIMENTS}

\section{A. Roughness discrimination}

In practice, the reflected Stokes vector $\boldsymbol{S}^{\prime}$ can only be measured for a limited number of discrete illumination angles $\left(\theta_{\mathrm{S}}, \varphi_{\mathrm{S}}\right)$ (see fig. 2) . This process can be considered as a sampling of the illumination space. In an attempt to minimize the number of images taken under different elevation angles, Lindner et al. [7] have proposed a method to know how many sampling points are necessary to measure the reflected intensity signal with a predetermined accuracy. To this end they used Shannon's sampling theorem. They found that the number of samples, i.e. the number of images in the illumination series must be larger than $2 / \sigma$. So to extract the reflection features from the reflected Stokes vector $\boldsymbol{S}^{\prime}$ with sufficient accuracy, a prior knowledge can be derived from former image series taken from similar objects or it can be estimated by an algorithm which performs an adaptive sampling of the illumination space.

Firstly, the proposed rough surface imaging algorithm has been tested. For this, we have chosen to observe a scene where two objects with different roughness are lying on a black sample holder (see fig.3).

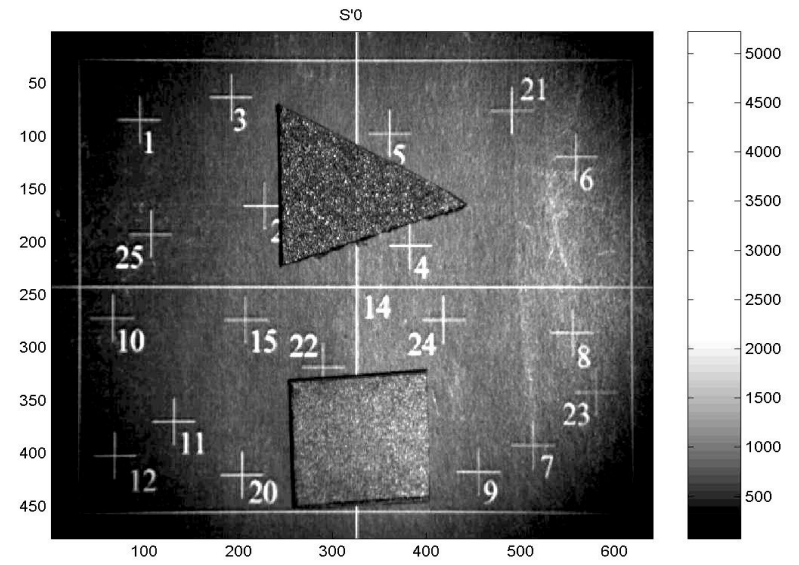

Fig.3: The observed scene (two objects with different roughness) 
These objects have the particularity to be planar so the two surfaces are oriented in the direction of the sample holder and therefore it is not necessary to use a stereovision algorithm to estimate their orientations. Furthermore these objects are made with the same plastic material of a part of electronic equipment cases, so the refractive indexes estimated by the proposed algorithm should be the same for the two objects.

Beforehand, we have measured the roughness (Ra parameter) of these objects with a portable roughness tester RT-10 of SM S. L. (resolution $0.01 / 0.04 \mathrm{~mm}$ ). The triangle object was the rougher one, with a measured value of $\mathrm{Ra}=5.87$, and the measured value for the square object was $\mathrm{Ra}=3.56$.

Next, we have made several measurements of the Stokes vector $\boldsymbol{S}^{\prime}$ of the reflected optical light wave for 10 elevation angles $\theta_{\mathrm{S}}$ of the light source (see fig.2). To this end the source is moved over the samples along a semicircular arc, yielding a sampling of the elevation angle in the interval $\left[0^{\circ}, 90^{\circ}\right]$. The illumination direction, the direction of observation and the surface normal are in-plan $\left(\varphi_{N}=\varphi_{\mathrm{S}}=90^{\circ}\right)$. Fig. 4 shows the Stokes images series for which the angle of observation (between the viewing direction and the surface normal) was $\theta_{\mathrm{N}}=0^{\circ}$.

These measurements were then used to estimate the refractive index for each pixel of the observed scene according to eq. (15). The mean values of estimated indexes were 1.72 with a standard deviation of 0.03 for the triangle, 1.71 with a standard deviation of 0.02 for the square, and 2.04 with a standard deviation of 0.04 for the sample holder. One can notice that these values are approximately the same for the two objects that is consistent with the fact that the two pieces are in the same material. But these values seem to be over-estimated in regard to the classical optical index value of plastic materials $(1.3<\eta<1.7)$. 


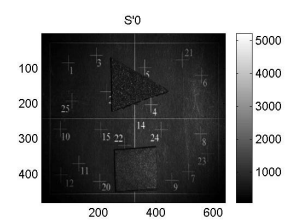

$$
\left(\theta_{S}=20^{\circ}\right)
$$
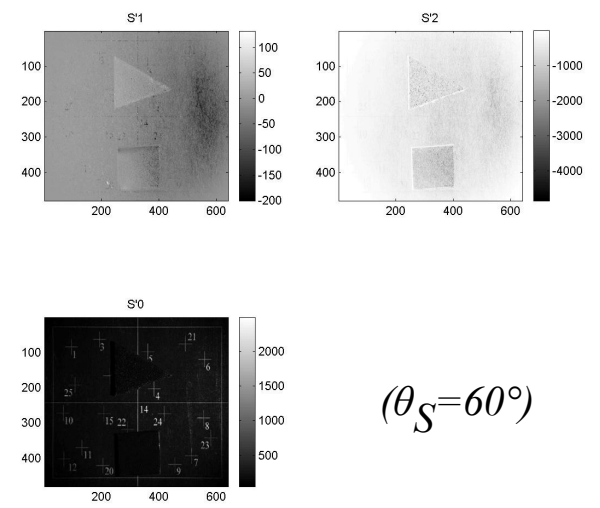

$$
\left(\theta_{S}=60^{\circ}\right)
$$
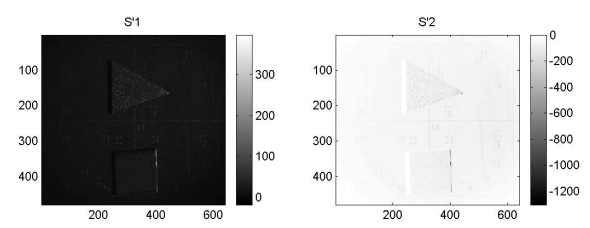

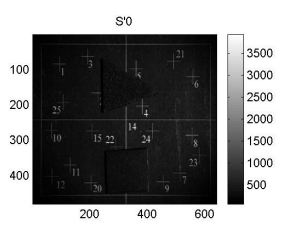

$$
\left(\theta_{S}=40^{\circ}\right)
$$
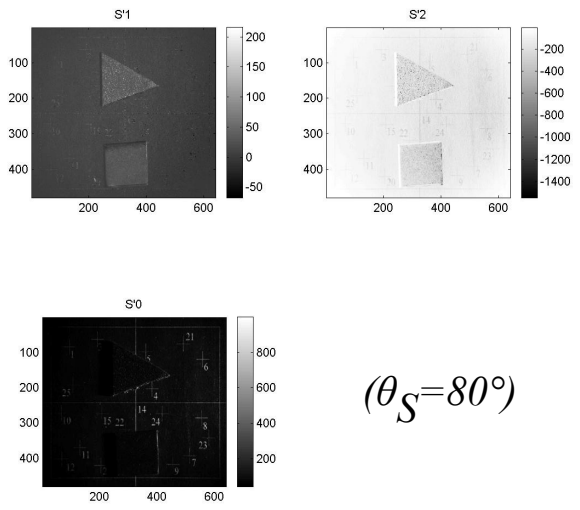

$$
\left(\theta_{S}=80^{\circ}\right)
$$

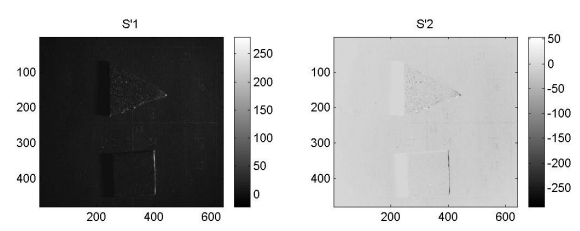

Fig.4: Stokes images for 4 elevation angles $\theta_{S}$ of the light source

These indexes $\eta$ were then used to compute the coefficients $M_{i j}$ for each position of the light source and to estimate the parameter $\sigma$ (according eq. (16-19)) for each pixel of the imaged scene.

Fig. 5 and 6 show an example of the two series of points computed with the measured Stokes parameters $\left(\mathrm{S}_{1 \mathrm{~S}}^{\prime}, \mathrm{S}_{2 \mathrm{~S}}\right)$ and the associated Mueller coefficients $\left(\mathrm{M}_{21}, \mathrm{M}_{33}\right)$. A line is then fitted on these $2 \mathrm{~N}$ points ( $\mathrm{N}$ positions of the light source). The slope is directly connected to roughness parameter $\sigma$ by equation $(16)\left(\right.$ slope $\left.=-1 / \sigma^{2}\right)$.

The average values of $\sigma$ are estimated from the pixels of the two regions of interest (square and triangle). The results are 0.268 with a standard deviation of 0.032 for the triangle and 0.221 with a standard deviation of 0.012 for the square. As expected the estimated values of 
$\sigma$ verify the sampling relation proposed by Lindner et al. [7] $(\sigma>0.2$ for ten positions of the light source).

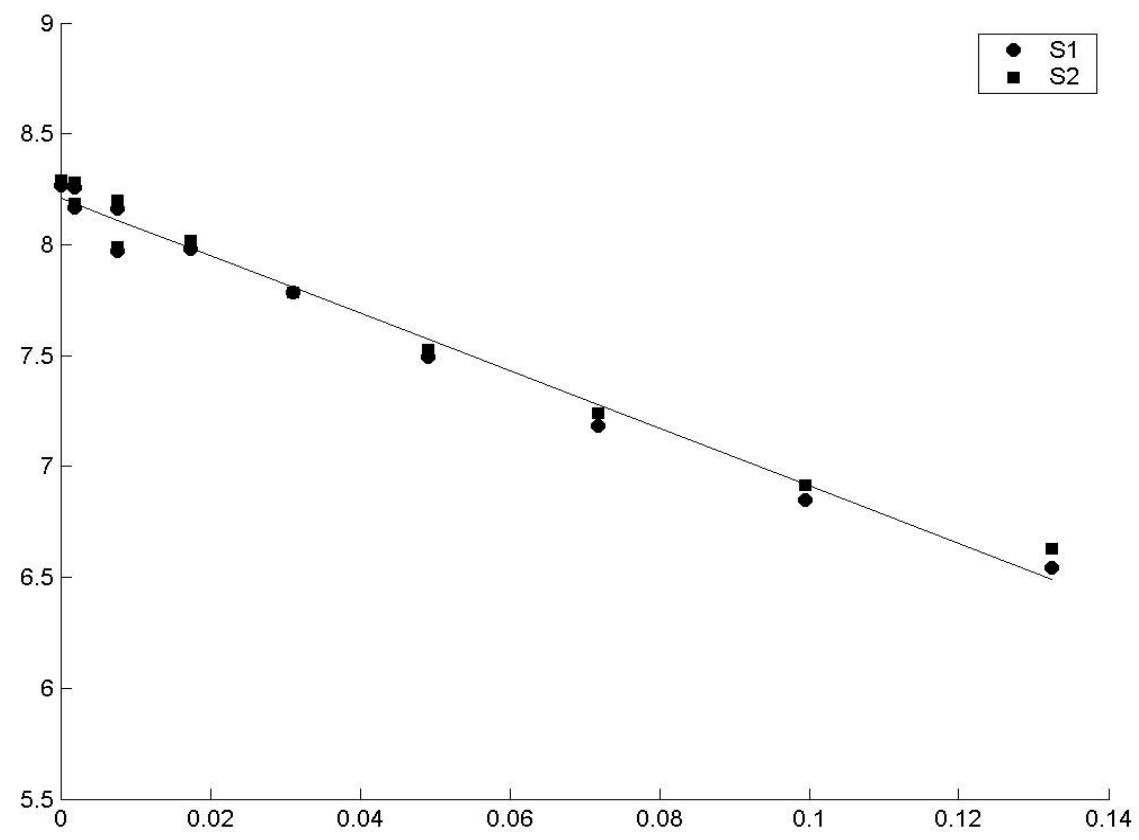

Fig.5 : Linear regression applied on the two series of points computed for an arbitrary pixel of the triangle region

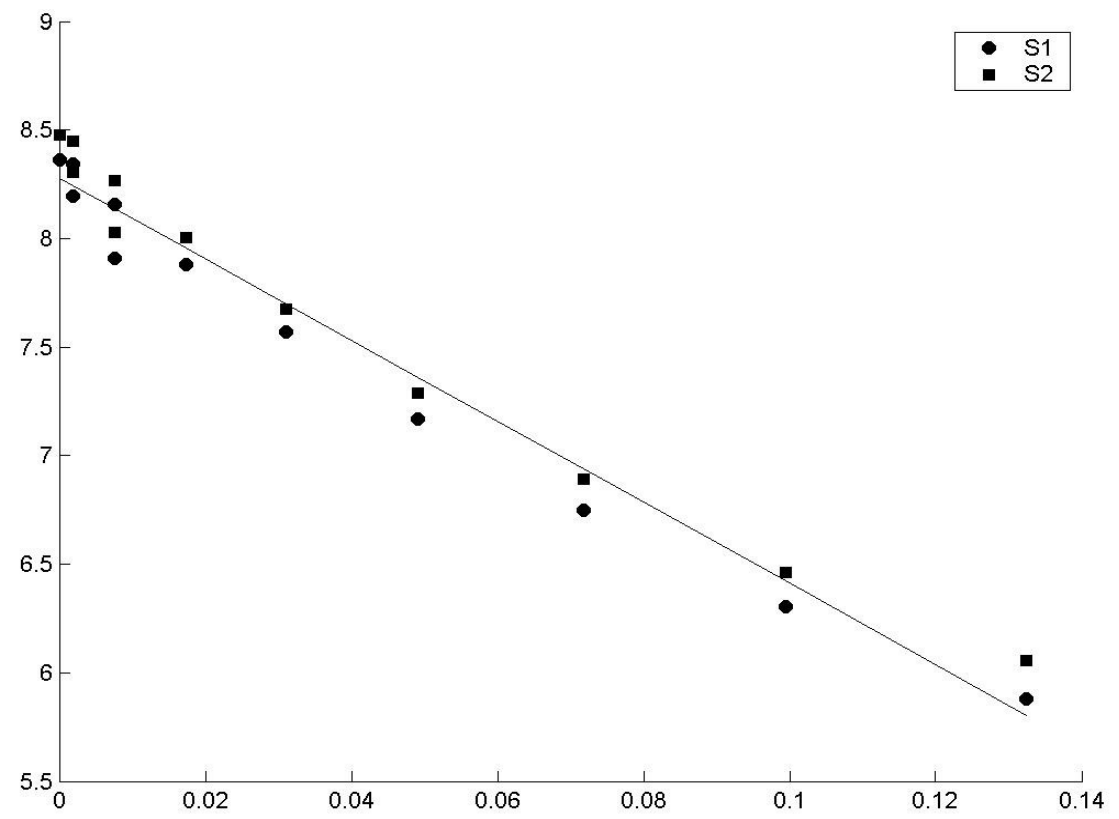

Fig. 6: Linear regression applied on the two series of points computed for an arbitrary pixel of the square region 
One can notice that the average value of $\sigma$ is larger for the triangle, which is the rougher object. Nevertheless, these values may not be regard as exact values of roughness but may be used as a roughness comparison criterion between two surfaces.

In a second experiment, we used the proposed algorithm to segment a textured surface according to its roughness. The application could be in the domain of quality control, for example to detect defects on varnished wood, like it is mentioned by Lindner et al. [7] where the defects originate from a lack of varnish or from impurities. Lindner obtained good results by estimating the local degree of specularity of the surface. Nevertheless, as it was previously mentioned, the proposed polarimetric method does not require any assumption on the diffuse component of reflection, this can improve defects detection. Moreover, the proposed method provides an image of the locally estimated refractive index. This additional information can also improved segmentation performance if varnish and wood have a different refractive index. Fig. 7 and 8 present results obtained with the proposed polarimetric method. The observed object (fig. 7 is a varnished piece of wood with a triangle area in which no varnish was applied (defect). Fig. 8 corresponds to the map of $\sigma$ parameter.

As we can see the $\sigma$ parameter is greater in the triangle region where no varnish was applied (the mean value is 0.011 for the varnished region and 0.024 for the not varnished wood). It is therefore possible to automatically detect effects by using a threshold. 


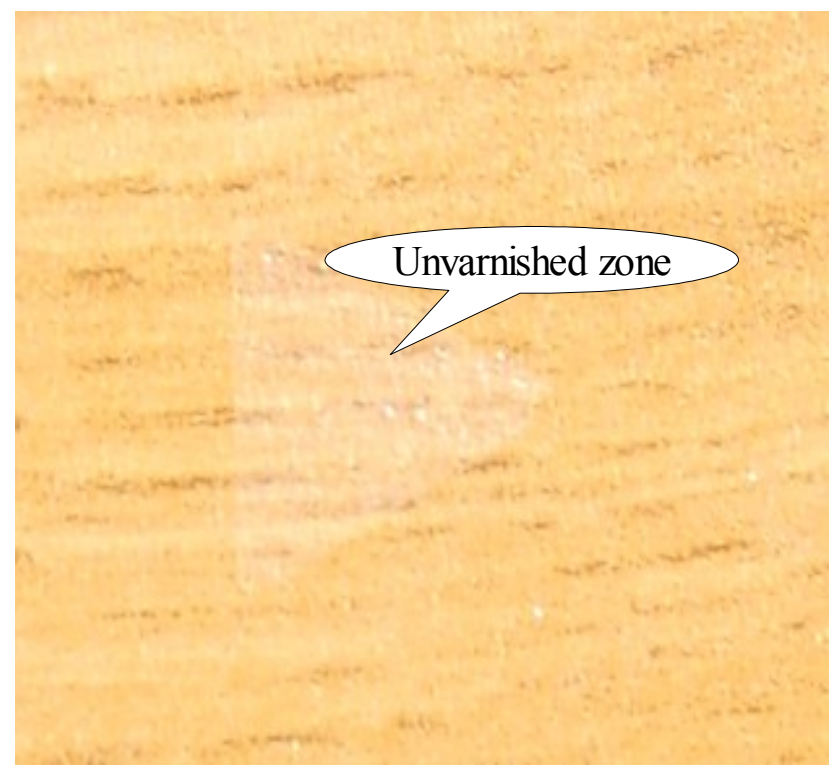

Fig.7: Intensity image of the observed piece of wood with histogram equalization for contrast enhancement

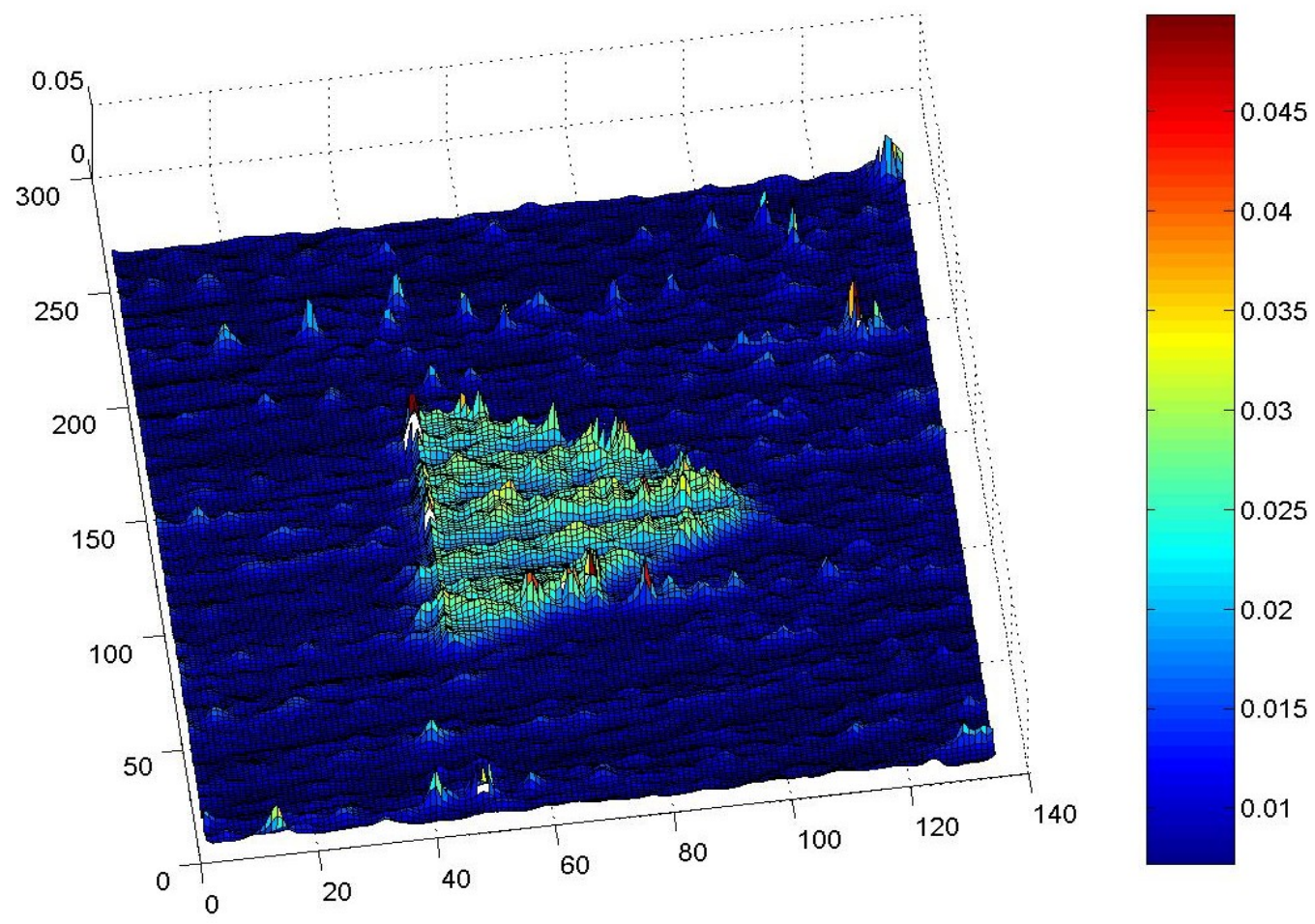

Fig.8: Estimated $\sigma$ value map for the observed scene 


\section{B. Metal-dielectric discrimination}

On the other hand, in particular case, where the varnished object is metallic, the polarimetric method allows us to detect and segment automatically regions which have a lack of varnish. In this particular application, the experimental process can be substantially simplified since it is not necessary to make several measurements of the reflected optical light wave Stokes vector $\boldsymbol{S}^{\prime}$ for various positions of the light source. Only one position is required. For this, we use the ellipsometric parameters $\tan (\psi)$ and $\Delta$ that correspond respectively to the modulus and phase of the Fresnel coefficients ratio:

$$
\frac{\rho_{P}}{\rho_{S}}=\tan \Psi e^{i \Delta}
$$

Substituting (20) into (7), we find that the Mueller matrix relation between the Stokes vector of the specular reflected and incident beams can be written as:

$$
M=\frac{\left|\rho_{S}\right|^{2}}{2}\left[\begin{array}{cccc}
1+\tan ^{2} \Psi & 1-\tan ^{2} \Psi & 0 & 0 \\
1-\tan ^{2} \Psi & 1+\tan ^{2} \Psi & 0 & 0 \\
0 & 0 & 2 \tan \Psi \cos \Delta & -2 \tan \Psi \sin \Delta \\
0 & 0 & 2 \tan \Psi \sin \Delta & 2 \tan \Psi \cos \Delta
\end{array}\right]
$$

According to eq. (12) and (13) we see that the ratio between the measured Stokes vector components $\mathrm{S}_{3}^{\prime}$ and $\mathrm{S}_{2}^{\prime}$ yields, for a linear $+45^{\circ}$ polarized light source:

$$
\frac{S_{3}^{\prime}}{S_{2}^{\prime}}=\frac{M_{43}}{M_{33}}=\frac{2 \tan \Psi \sin \Delta}{2 \tan \Psi \cos \Delta}=\tan \Delta
$$


So the parameter $\Delta$ may be estimated by the polarimetric Stokes measurements and may bring information for material classification [21].

Indeed, in the case of a metallic object, the Fresnel coefficients are complex numbers and the phase $\Delta$ (eq. 20) is not null. On the other hand, for dielectric surface like varnished surface, the Fresnel coefficients are real numbers and so $\Delta$ is null. It is then possible to distinguish varnished regions on metallic objects like it is illustrated fig.9,10 and 11.

For the example presented Fig.10 we used a lacquer for hair because the deposit has the particularity to be invisible on the intensity image whereas the text written with the lacquer is clearly visible in the $\Delta$ image.

As we can see on these three examples the $\Delta$ image obtained by the polarimetric measurements may be used to detect either lack or residue of dielectric material on a metallic surface.

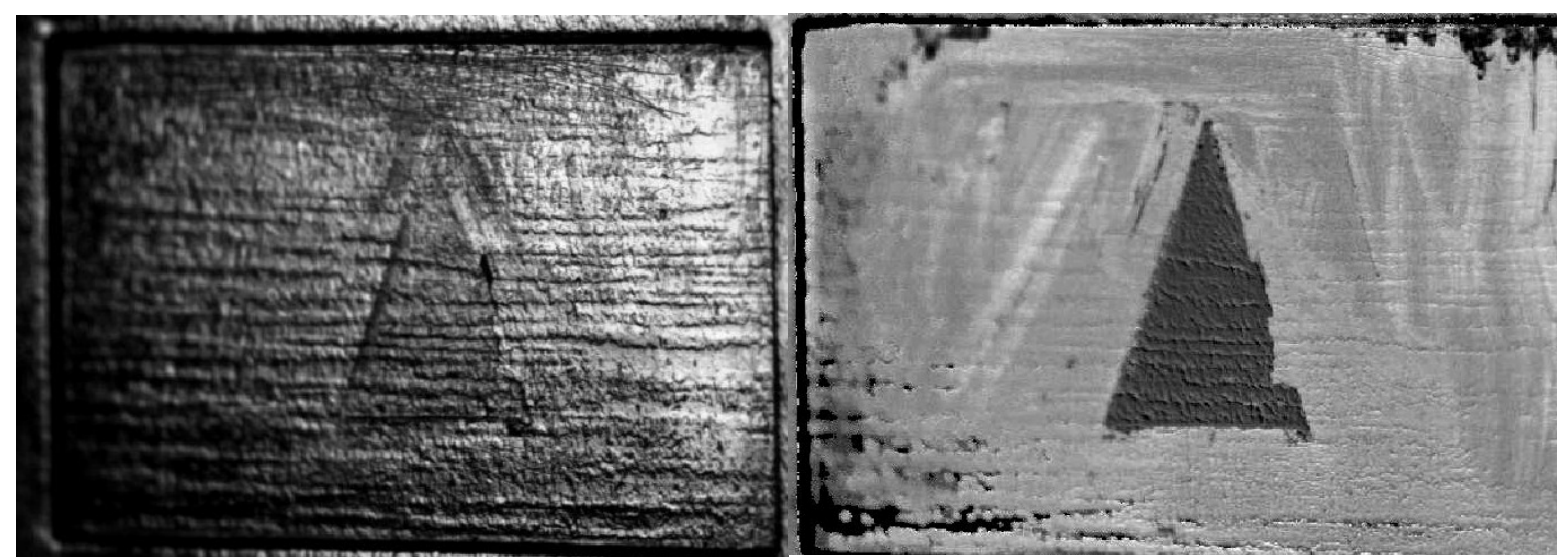

Fig.9: Detection of varnished defect (triangle) on a metallic object (varnish for automobile painting is used) left: intensity image with histogram equalization for contrast enhancement; right $\triangle$ image 


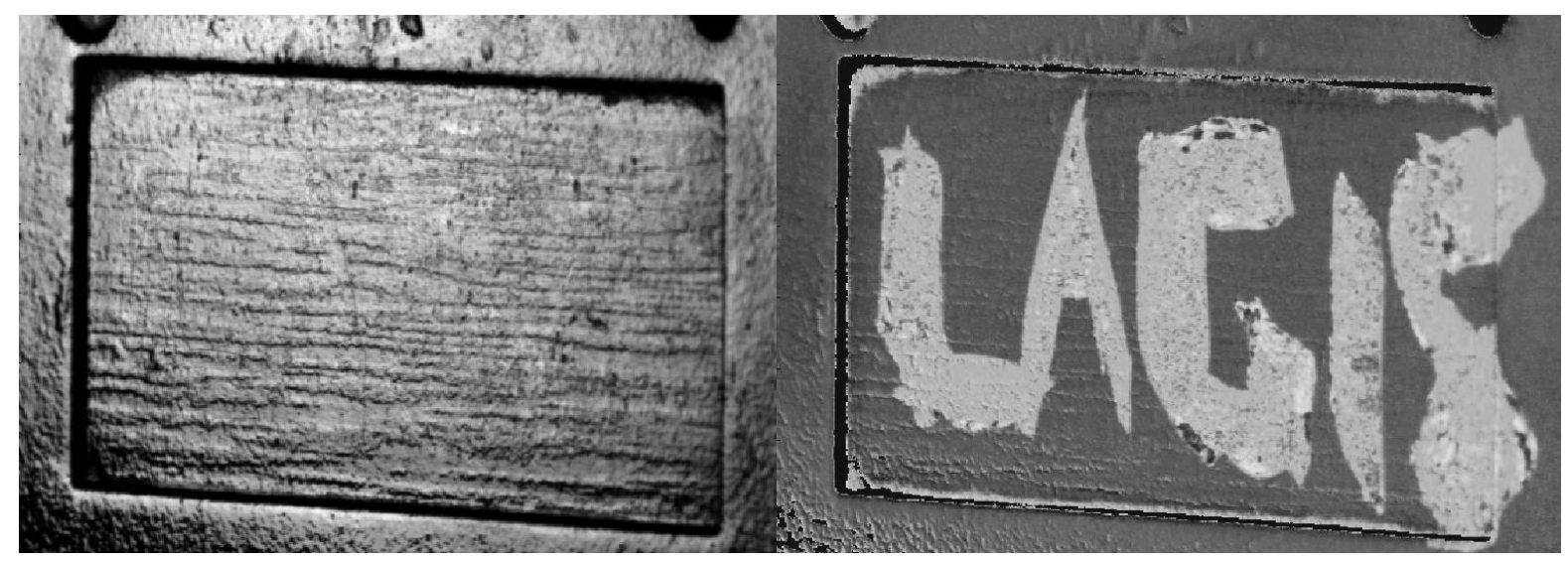

Fig. 10: Detection of thin dielectric deposit (text) on a metallic object (the text was written with a lacquer for hair) left: intensity image with histogram equalization for contrast enhancement; right $\Delta$ image

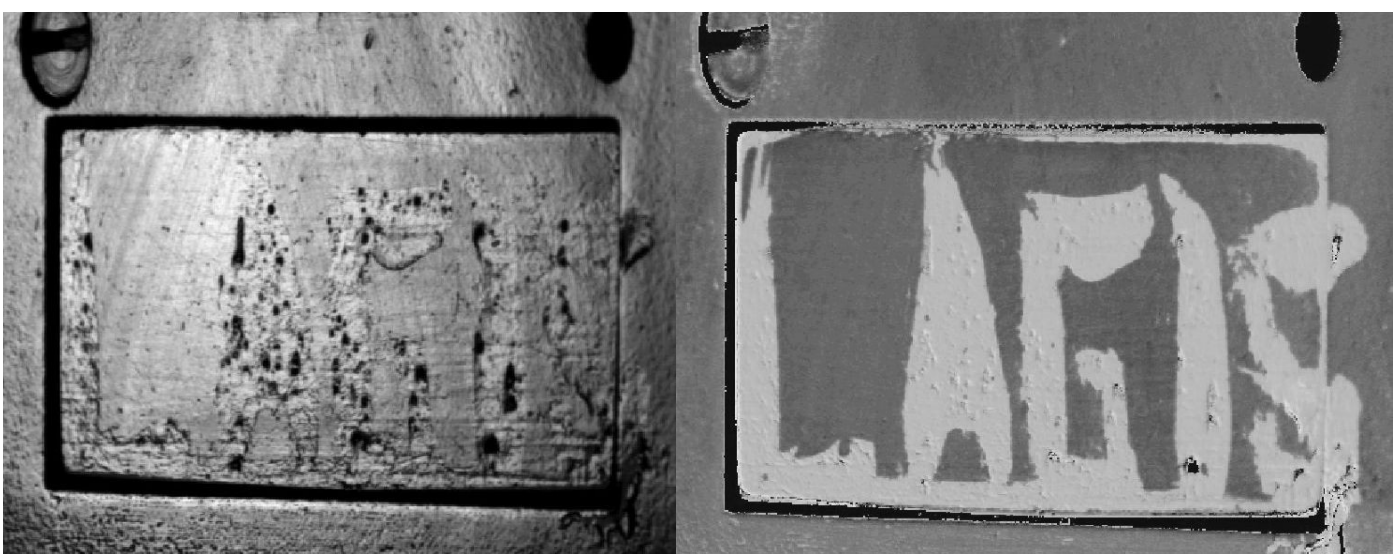

Fig. 11: Detection of oil traces (text) on a metallic object (the text was written with lubrication oil for industrial tools) left: intensity image with histogram equalization for contrast enhancement; right $\Delta$ image 


\section{CONCLUSION}

A polarimetric vision system yielding a pixel estimation of surface roughness was presented. The proposed technique assumes object surfaces are made up of a collection of small facets much larger than the imaging wavelength. Therefore, the reflection model assumes that the reflected light is a linear combination of a diffuse reflection component and a specular one, depending on the probability density function of microfacets orientations related to the surface roughness. The advantage of the polarimetric approach is that it allows us to estimate the roughness parameter without assumption on the model of the diffuse component. Diffuse-specular separation is not required either .

The proposed non-contact method does not require expensive or large equipment, but simply a Stokes polarimeter. Furthermore, it allows us to obtain an image of the observed scene for which the roughness and the refractive index of the observed objects are estimated at each pixel. This work has potential application to inspection problems in industrial environment, for example to detect defects on varnished objects. From this proposed measurement device, automatic systems of control and classification using conjointly roughness and refractive index will be addressed in future works. 


\section{APPENDIX}

\section{Spherical trigonometry:}

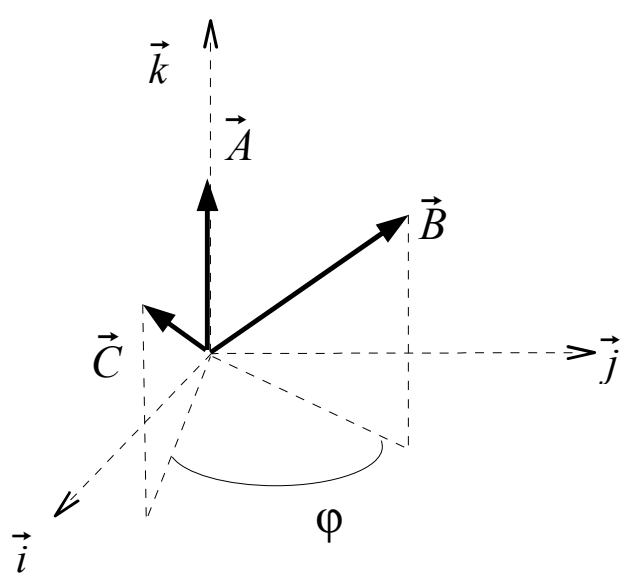

Let $a$ denote the angle between vectors $\vec{B}$ and $\vec{C}, b$ the angle between vectors $\vec{A}$ and $\vec{C}$ and $c$ the angle between vectors $\vec{A}$ and $\vec{B}$.

These three angles are related by the fundamental spherical trigonometric relation:

$$
\cos a=\cos c \cos b+\sin c \sin b \cos \varphi
$$

In the case of the proposed measurement device all angles are defined using the viewing direction for reference. Therefore, the model input parameters are the positions of the light source $\left(\theta_{\mathrm{S}}, \varphi_{\mathrm{S}}\right)$ and of the observed $\operatorname{surfaces}\left(\theta_{\mathrm{N}}, \varphi_{\mathrm{N}}\right)$ (orientation of the normal vector). When the light beam is reflected by a planar microfacet, the angle $\theta_{\mathrm{i}}^{\prime}$ between the incident light ray and the facet's normal, which is equal to the angle between the facet's normal and the reflected light ray, is given by: 


$$
\theta^{\prime}=\frac{\theta_{S}}{2}
$$

The others parameters of eq. (1) $\left(\theta_{\mathrm{R}}, \theta_{\mathrm{i}}\right.$ and $\left.\alpha\right)$ are obtained from $\left(\theta_{\mathrm{S}}, \varphi_{\mathrm{S}}\right)$ and $\left(\theta_{\mathrm{N}}, \varphi_{\mathrm{N}}\right)$ using:

- $\theta_{R}=\theta_{N}$

- If $\vec{A}$ represents the reflected light ray, $\vec{B}$ the global normal to the surface and $\vec{C}$ the incident light ray we have $a=\theta_{\mathrm{i}}, b=\theta_{\mathrm{S}}$ and $c=\theta_{\mathrm{N}}$. So, the angle $\theta \mathrm{i}$ can be estimated by the fundamental relation:

$$
\cos \theta_{i}=\cos \theta_{N} \cos \theta_{S}+\sin \theta_{N} \sin \theta_{S} \cos \left(\varphi_{S}-\varphi_{N}\right)
$$

- The same relation is used for determining the angle $\alpha$ between the local microfacet's normal and the global normal:

$$
\cos \alpha=\cos \theta_{N} \cos \frac{\theta_{S}}{2}+\sin \theta_{N} \sin \frac{\theta_{S}}{2} \cos \left(\varphi_{S}-\varphi_{N}\right)
$$




\section{REFERENCES}

[1] D. J. Whitehouse, "Surface metrology ", Meas. Sci. Technol., Vol. 8, pp. 955-972, 1997

[2] J. Liu, K. Ymazaki, Y. Zhou, "A reflective fiber optic sensor for surface roughness inprocess measurement", Journal of Manufacturing Science and Engineering, Vol. 124, pp. 515522,2002

[3] S. Wang, Y. Tian, C. J. Tay and C. Quan, "Development of a laser-scattering-based probe for on-line measurement of surface roughness", Applied Optics, Vol. 42(7), pp. 13181324,2003

[4] M. E. McKnight, T. V. Vorburger, E. Marx, M. E. Nadal, P. Y. Barnes, "Measurements and predictions of light scattering by clear coating ", Applied optics, Vol. 40(13), pp. 2159-2168, 2001

[5] F. Solomon, K. Ikeuchi, "Extracting the shape and roughness of specular lobe objects using four light photometric stereo", IEEE, Trans. on Pattern analysis and machine inte., Vol. 18(4), pp. 449-454, 1996

[6] D. Miyazaki, R. T. Tan, K. Hara, K. Ikeucji, "Polarization-based inverse rendering from a single view ", Proc. IEEE International conference on computer vi, Vol. 2, pp. 982987,2003

[7] C. Lindner, F. Puente Leon, "Reflection-based surface segmentation using active illumination ", Proc. IMTC 2006 Instrum. and Meas Tech. Conf., Vol. , pp. 157-162, 2006 [8] R. L. Cook and K. E. Torrance, "A reflectance model for computer graphics ", Computer Graphics, Vol. 15(3), pp. 307-316, 1981

[9] K. E. Torrance and E. M. Sparrow, "Theory for off-specular reflection from roughened surfaces ", Journal of the Optical Society of America, Vol. 57, pp. 1105-1114, 1967 
[10] M. Oren, S. K. Nayar, "Generalization of the Lambertian model and implications for machines vision ", Int. J. Comput. Vision, Vol. 14(3), pp. 227-251, 1995

[11] S. Shafer, "Using color to separate reflection components ", Color Res. Applications, Vol. 10(4), pp. 210-218, 1985

[12] S. Lin, Y. Li, S. B. Kang, X. Tong and H. Shum, "Diffuse-Specular separation and depth recovery from image sequences ", Proc. European Conference on Computer Vision, Vol. 3, pp. 89-103, 2002

[13] D. Kim, S. Lin, K. Hong, H. Shum, "Variational separation using color and polarization ", Proc. IAPR Workshop on machine Vision and Appli., Vol. , pp. 176-179, 2002

[14] S. K. Nayar, X. Fang and T. Boult, "Separation of reflection components using color and polarization ", International Journal of Computer Vision, Vol. 21(3), pp. 163-186, 1997

[15] L. B. Wolff and T. E. Boult, "Constraining object features using a polarization reflectance model ", IEEE Trans. On Pattern Analysis and Machine Intell, Vol. 13(7), pp. $635-657,1991$

[16] F. Goudail, P. Terrier, Y Takakura, L. Bigué, F. Galland and V. Devlaminck, "Target Detection with a liquid crystal-based passive Stokes polarimeter ", Applied Optics, Vol. 43(2), pp. 274-282, 2004

[17] J. S. Tyo, "Design of optimal polarimeters: maximization of SNR and minimization of systematic errors", Appl. Opt., Vol. 41, pp. 619-630, 2002

[18] E. Collet, "Polarized light: fundamentals and applications", Marcel Dekker, 1993

[19] R. M. A. Azzam, N. M. Bashara, "Ellipsometry and polarized light", North-Holland personal library, 1999

[20] K. N. Kutulakos, E. Steger, "A theory of refractive and specular 3D shape by lightpath triangulation ", Proc. ICCV'05, Vol. 2, pp. 1448-1455, 2005 
[21] H. C. Chen, L. B. Wolff, "Polarization phased-based method for material classification in computer vision", International. J. of Computer, Vis., Vol. 28(1), pp. 73-83, 1998 